\title{
Erfahrungen mit den Qualitätsindikatoren in der Schweiz
}

\section{Ausgangslage \\ $\nabla$}

Seit dem Jahr 2009 werden in der Schweiz Qualitätsindikatoren basierend auf Routinedaten publiziert [1]. Die Publikation erfolgt durch das Schweizerische Bundesamt für Gesundheit (BAG) auf der gesetzlichen Grundlage des Krankenversicherungsgesetzes (KVG, Art. 22a).

\section{Erste Phase: Skandalisierung und kollektive Ablehnung \\ $\checkmark$}

Die ersten Reaktionen auf die Publikation waren heftig und die Kritik am zuständigen Bundesamt ausgesprochen stark. Dabei waren sich die Standesvertreter der Ärzteschaft, der Krankenhäuser und der Patienten einig. Die Analyse von Sterberaten für Qualitätszwecke wurde als nicht valide bezeichnet, bis hin $\mathrm{zu}$ „nicht mit den ethischen Grundsätzen der medizinischen Wissenschaften vereinbar“. Eine typische Aussage in diesem $\mathrm{Zu}-$ sammenhang war auch: „Bei uns dürfen die Patienten sterben.“

Seither befindet sich die Schweizer Q-Szene im Umbruch. Unter anderem ging daraus der Nationale Verein für Qualitätsentwicklung in Spitälern und Kliniken (ANQ) als Zusammenschluss von verschiedenen bestehenden Organisationen hervor. Die Aktivitäten des ANQ fokussieren zum aktuellen Zeitpunkt auf die Definition von nationalen Messungen zu den Themen Sturz, Dekubitus, nosokomiale Infektionen und Patientenzufriedenheit.

\section{Zweite Phase: Potenzial erkannt und aufkeimende Nutzung \\ $\checkmark$}

Einzelne Leistungserbringer und vereinzelt die Gesundheitsdirektionen der Kantone haben das Potenzial des Qualitätsmanagements mit Routinedaten erkannt und setzen die Indikatoren für ein Screening der Leistungserbringung und für die nachhaltige Stimulierung des internen Verbesserungsprozesses ein.

Von Beginn an wurde das Projekt auch von den Universitätsspitälern konstruktiv unterstützt. So waren es die Universitätsspitäler Basel und Zürich, die als Erste ihre Resultate publiziert haben. Im Rahmen einer Arbeitsgruppe der Universitätsspitäler wurden Vorschläge für die Weiterentwicklung der Indikatoren erarbeitet, die teilweise auch in die G-IQIs übernommen wurden.
Das Inselspital Universitätsspital Bern ist seit 2010 Mitglied der Initiative Qualitätsmedizin $\left(\mathrm{IQ}^{\mathrm{M}}\right)$. Im April 2011 wurde an der Universitätsklinik für Intensivmedizin das erste Peer Review nach dem Muster der IQ ${ }^{\mathrm{M}}$ in der Schweiz durchgeführt.

\section{Ausblick}

Qualitätsmanagement mit Routinedaten wird im Zuge der anstehenden Entwicklungen in der Schweiz, insbesondere der Einführung des neuen Finanzierungssystems im stationären Bereich (Swiss DRG) zweifellos an Gewicht gewinnen.

Angesichts der verpflichtenden Publikation der Swiss Inpatient Quality Indicators (CH-IQIs) für sämtliche Akutkrankenhäuser ist die Schweiz vergleichsweise weit fortgeschritten. Die reine Darstellung der Resultate, wie sie im Moment betrieben wird, ist aber erst der Ausgangspunkt für den kontinuierlichen Verbesserungsprozess.

Aufgrund der abgestimmten Systeme der German (G-IQI), Austrian (A-IQI) und Swiss Inpatient Quality Indicators (CH-IQI) ist es ansatzweise möglich, Vergleiche zwischen den drei Ländern zu machen. Dies entspricht dem Trend in Richtung Patientenfreizügigkeit, der sich in Europa abzeichnet. In der Europäischen Union wurden unlängst die entsprechenden Rahmenbedingungen in der Direktive 2011/24 festgelegt [2]. Die europäischen Staaten haben bis Oktober 2013 Zeit, die Regelungen in ihre nationalen Gesetzgebungen zu übernehmen. Die Schweiz wird diese im Zuge der Acquis communautaire ebenfalls übernehmen müssen.

Autorenerklärung: Der Autor erklärt, dass keine relevanten finanziellen Verbindungen in Bezug auf dieses Manuskript bestehen.

\section{Literatur}

1 Qualitätsindikatoren der Schweizer Akutspitäler (Schweizerisches Bundesamt für Gesundheit BAG, 2009, 2010)

2 Richtlinie 2011/24/EU des europäischen Parlaments und des Rates vom 9. März 2011 über die Ausübung der Patientenrechte in der grenzüberschreitenden Gesundheitsversorgung
D. W. Zahnd

Qualitätsmanagement

Schlüisselwörter $\checkmark$ Qualitätsmanagement Qualitätsindikatoren Routinedaten

Schweiz

Keywords

quality management quality indicators administrative data Switzerland Inselspital Universitätsspital Bern, Schweiz

Bibliografie

Dol 10.1055/s-0031-1286078 Dtsch Med Wochenschr 2011; 136: S49 - (c) Georg Thieme Verlag KG Stuttgart - New York . ISSN 0012-0472

Korrespondenz

Dr. phil. Daniel W. Zahnd

Inselspital Universitätsspital Bern

Ärztliche Direktion FQM

3010 Bern

Schweiz

Tel. +41316329577

eMail daniel.zahnd@insel.ch 